\title{
Molecular characterization of a cryptic wheat-Thinopyrum intermedium translocation line: evidence for genomic instability in nascent allopolyploid and aneuploid lines
}

\author{
Yingshan Dong ${ }^{1,2}$, Xiuling $\mathrm{Bu}^{1}$, Yushi Luan ${ }^{3}$, Mengyuan $\mathrm{He}^{1}$ and Bao Liu ${ }^{1}$ \\ ${ }^{1}$ Northeast Normal University, Institute of Genetics \& Cytology, Changchun 130024, China. \\ ${ }^{2}$ National Center for Transgenic Plant Research \& Commercialization, Jilin Academy of Agricultural \\ Sciences, Jilin, China. \\ ${ }^{3}$ Dalian University of Technology, Department of Bioengineering, Dalian 116012, China.
}

\begin{abstract}
A putative translocation line (\#32), together with a disomic addition line (TAI27) and an octo-amphiploid line (Zhong3) of common wheat and Thinopyrum intermedium were characterized by restriction fragment length polymorphism (RFLP) analysis, using probes covering all seven homoeologous groups of Triticeae. Line 32 was confirmed to be a cryptic translocation line, based on the detection of multiple introgressed hybridization fragments specific to $T h$. intermedium in the RFLP patterns, and the absence of a hybridization signal in GISH analysis. In addition, extensive genomic changes, as compared to the wheat parent, were detected on all three lines studied, with a great majority of changes showing concordance among the lines. Our data is consistent with the emerging view that nascent allopolyploid and aneuploid plant genomes are highly dynamic, which may generate novel introgressed materials for breeding.
\end{abstract}

Key words: translocation, genomic changes, allopolyploidy and aneuploidy, wheat, Thinopyrum intermedium.

Received: July 1, 2003; Revised: November 21, 2003; Accepted: November 27, 2003.

\section{Introduction}

Introgression of desirable traits from wild relatives to cultivated crops by means of wide hybridization has been an exceptionally successful practice in crop improvement. A typical example of alien gene transfer is the generation of cytologically discernible translocation lines. However, in some cases, traits of interest are transferred to the recipient crop without cytological changes in its genomic constitution (e.g., Multani et al., 1994), thus implying that the introgressed chromatin segments are cryptic. This type of introgression is "atypical" in that it is often produced without apparent homoeologous chromosome recombinations (Brar and Khush, 1997). Although the mechanism for the occurrence of such cryptic translocations is unknown, their occurrence seems frequent, as evidenced by molecular marker-assisted QTL analysis (Zamir, 2001). Moreover, the introgressed materials are likely to be the most useful in terms of breeding, because the likelihood of dragging in of unwanted traits from the wild species is minimized. Therefore, it is of significance to characterize such materials,

Send correspondence to Bao Liu. Northeast Normal University, Institute of Genetics \& Cytology, Changchun 130024, China. E-mail: baoliu@nenu.edu.cn. both for the purpose of their fuller utilization in breeding programs and of providing clues to the mechanism for their genesis.

Thinopyrum intermedium (Host) Barkworth and Dewey, syn. Agropyron intermedium (Host) P. B., is a hexaploid species (genomes, JJJsJsSS, $2 n=6 x=42$ ) that possesses numerous traits that are important for wheat improvement. Various partial amphiploids $(2 \mathrm{n}=8 \mathrm{x}=56)$ containing the entire genomes of common wheat (AABBDD) and 14 chromosomes of Th. intermedium have been produced, and several sets of disomic alien addition lines are also currently available. (He et al.,1988; Larkin et al., 1995). In addition, translocation lines that retain the traits of interest, such as resistance to rust, to wheat streak mosaic virus (WSMV) and to barley dwarf virus (BYDV), have been produced by spontaneous translocation, irradiation and tissue culture (Banks et al., 1995; Wang and Zhang, 1996; Han et al., 1998; Crasta et al., 2000; Liu et al., 2001; Zhang et al., 2001). As expected, most of these analyzed materials were found to be typical translocation lines, i.e., to contain cytologically discernible chromosomal segments from Th. intermedium, as revealed by chromosome banding techniques and/or genomic in situ hybridization (GISH) 
(Wang and Zhang, 1996; Han et al., 1998; Crasta et al., 2000). During our work on characterization of interesting derivatives from amphiploids and addition lines of wheat and Th. intermedium, we found that, among typical translocation lines containing chromosomal segments translocated from Th. intermedium based on GISH analysis (e.g., Han et al., 1998), several lines are "atypical" in that no distinct chromosomal segment from Th. intermedium could be detected even by high resolution GISH analysis (Han et al., 1998 and unpublished data). Nevertheless, these lines, such as \#32, showed traits apparently derived from Th. intermedium, such as resistance to BYDV or rust, elevated protein percentage and tolerance to drought (our unpublished data). It thus seems likely that these lines are cryptic translocated materials containing minute introgressed Th. intermedium chromatins.

In the present communication, we characterize one of these lines (Line \#32) by restriction fragment length polymorphism (RFLP) analysis using mapped wheat homoeologous group-specific nuclear sequences (Gale et al., 1993) and Th. intermedium-enriched repeats as probes. We report that Line \#32 contains multiple introgressed hybridization fragments specific to Th. intermedium. Moreover, this line exhibits extensive genomic changes at the DNA level that are often concordant with changes in the amphiploid and/or addition line. Taken together, our results lend support to the emerging view that nascent plant polyploid and aneuploid genomes are intrinsically labile, often generating various non-Mendelian genetic and epigenetic changes.

\section{Material and Methods}

\section{Plant material}

An octoploid partial amphiploid (Zhong3) of common wheat (Triticum aestivum L.) and Thinopyrum intermedium (Host) Barkworth and Dewey, its wheat and Th. intermedium parental lines, a disomic alien addition line (TAI27) derived from Zhong3, and a putative translocation line (\#32) derived from TAI27, were used in this study. Detailed information concerning production and cytological characterization of the amphiploid and addition lines was published previously (He et al., 1988).

\section{Cytological and RFLP analysis}

The chromosomal constitution of the above materials at the cytological level was examined by conventional cytological analysis on squashed root-tip cells. Zhong3, TAI27 and Line \#32 were found to have respectively 56, 44, and 42 normal-looking chromosomes, i.e., no gross chromosomal aberration was observed. In addition, GISH analysis (exactly as in Han et al., 1998), even though confirmed the identity of Zhong3 (42 chromosomes of wheat and 14 chromosomes of Th. intermedium) and TAI27 (42 chromosomes of wheat and a pair of smaller chromosomes derived from Th. intermedium), revealed the absence in Line \#32 of Th. intermedium chromosomes or chromosomal segments (data not shown). The analyzed seeds from each plant material were germinated, and genomic DNA was isolated by the CTAB method from expanded leaves of pooled individuals. The DNA was digested with three restriction enzymes, BamHI, DraI, and EcoRI, fractionated in $0.8 \%$ agarose gel, and transferred onto Hybond $\mathrm{N}^{+}$nylon membranes (Amersham). Thirty-six mapped wheat RFLP probes covering all seven homoeologous chromosome groups of Triticeae (Gale et al., 1993), and two repeats isolated from genomic DNA of Th. intermedium, were radio-labeled with ${ }^{32} \mathrm{P}$ by the random primer method, and used as probes for Southern blot hybridization. Pre-hybridization, hybridization and post-hybridization washing were performed as described by Liu et al. (1997). Filters were exposed to X-ray films with an intensifying screen for 3-7 days at $-80{ }^{\circ} \mathrm{C}$.

\section{Results and Discussion}

The identity of the origin of the 14 chromosomes of Thinopyrum intermedium in Zhong3 and other wheat - Th. intermedium octoploids is controversial: some authors believe that they are constituents of a single, complete $T h$. intermedium genome (He et al., 1988), whereas others assume that they are from different Th. intermedium genomes, and have constituted a "synthetic" genome (Fedak et al., 2000; Chen et al., 2003). In the present work, of the mapped sequence/enzyme combinations (S/Es) analyzed, none of the six polymorphic S/Es, which mapped to the homoeologous group 1, detected the presence of $T h$. intermedium-specific hybridization fragments in Zhong3 (Table 1). This fact suggests that, assuming complete syntheny between the chromosomes of Th. intermedium and those of wheat, the 14 chromosomes in Zhong3 originated from Th. intermedium probably do not include the homoeologous group 1, therefore favoring the view that the 14 chromosomes do not belong to a single and complete genome (Fedak et al., 2000; Chen et al., 2003). We noted, however, that due to the extensive genomic restructuring or changes at the DNA level in the octoploid, as will be detailed below, it also is possible that the six homoeologous group $1 \mathrm{~S} /$ Es used may happen to be in those loci, being lost in Zhong3 (Table 1). Therefore, more $\mathrm{S} /$ Es from group 1 need to be analyzed in order to ascertain the absence or presence of Th. intermedium group 1 chromosome(s) in Zhong3.

Our earlier study using 12 wheat RFLP probes implied that the pair of structurally altered chromosomes (much smaller than normal chromosomes of either species) originated from Th. intermedium in the disomic addition line TAI27 was likely to be a recombinant of chromosome segments with homology to at least two homoeologous groups, 2 and 7 (Liu et al., 2001). In the present work, using more informative S/Es, we confirmed this hypothesis, and 


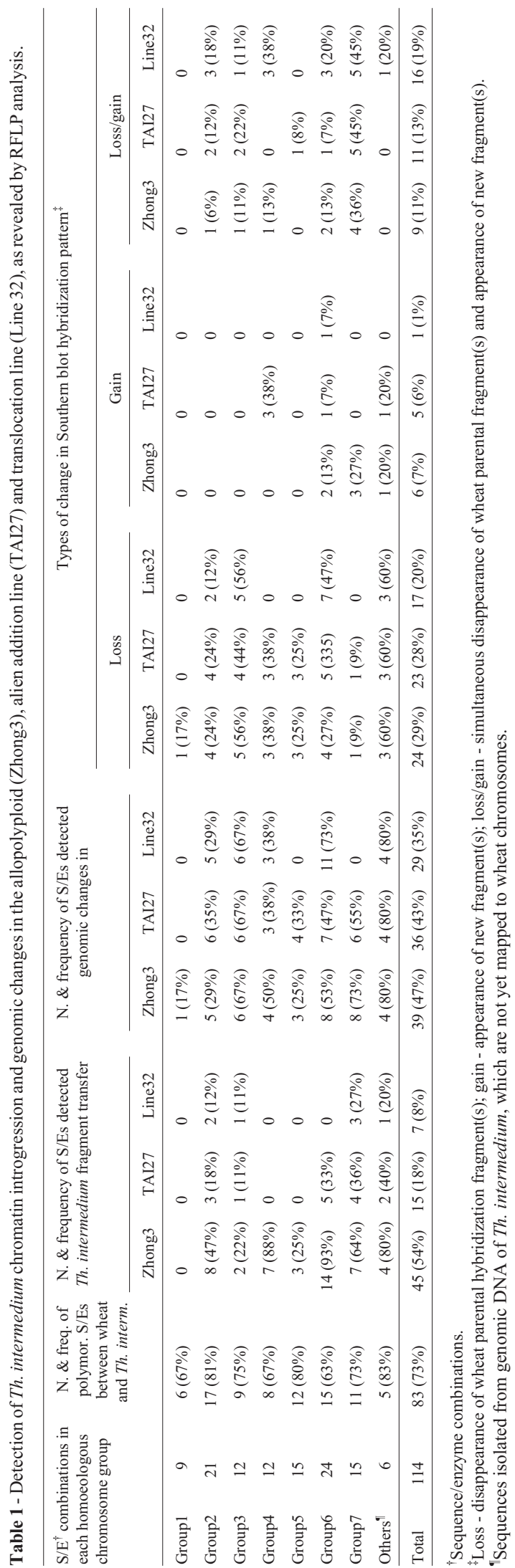

possibly extended the fragmental homology also to groups 3 and 6 (Table 1). However, differently from our earlier study (Liu et al., 2001), where a wheat plant "extracted" from TAI27 was used for direct comparison, in the present work only the immediate wheat parent of Zhong3 and TAI27 was included. Consequently, it cannot be ruled out that the detection in TAI27 of some of the Th. intermedium hybridization fragments (Table 1) was resultant from $T h$. intermedium chromatin segments integrated into wheat chromosomes, rather than from the pair of smaller chromosomes added. In fact, based on the present results (see below), it is most likely that such events indeed occurred. Notwithstanding the various possibilities, it seems safe to conclude that the pair of smaller chromosomes in TAI27 is a mosaic of chromosome segments originating from multiple Th. intermedium chromosomes.

GISH analysis having revealed complete absence of discrete hybridization signals denoting the existence of chromosomal segments from the wild species in Line \#32 (data not shown), it is surprising to note that nearly half of the polymorphic S/Es detecting Th. intermedium fragment transfer in TAI27 also detected the presence of $T h$. intermedium fragments in Line \#32 (Table 1 and Figures 1a, b, c, d, e). That the observed polymorphic hybridization fragments are not due to differential or incomplete DNA digestion in the various samples was ascertained by ethidium bromide staining on digested DNAs after agarose electrophoresis (Figure 1f), and by hybridization of the same set of blots to a single-copy cellular gene (encoding the putative sterol C24 methyltransferase, genbank accession: AY226581). (Figure 1g). Thus, the presence of Th. intermedium-specific fragments, together with phenotypic traits expressed by Line \#32, which are characteristic of the wild species, strongly suggests that Line \#32 contains multiple cryptic introgressed loci from Th. Intermedium, acquired prior to and/or in the process of formation of addition line TAI27. The fact that most of these introgressed fragments in Line \#32 and TAI27 also showed good concordance to those in amphiploid Zhong3 (Figures 1a, d, e and data not shown) indicates that the majority of the introgression events must have occurred at the amphiploid stage, followed by vertical transmission to the addition and translocation lines, with additional changes occurred at the addition line stage (e.g., Figures 1b, d). This implies instability of newly formed amphiploid and aneuploid genomes, as was indeed confirmed by comparing the Southern blot hybridization patterns of each of the amphiploid Zhong3, addition line TAI27 and translocation line \#32 with that of their wheat parent (Table 1 and Figure 1).

Since amphiploid Zhong 3 contains the entire genome of common wheat, it would be expected that Zhong3 per se and its direct derivatives retain all hybridization fragments of wheat and some fragments of Th. intermedium. Contrary to this expectation, polymorphic S/Es of all seven homoeologous groups detected genomic changes in amphiploid 


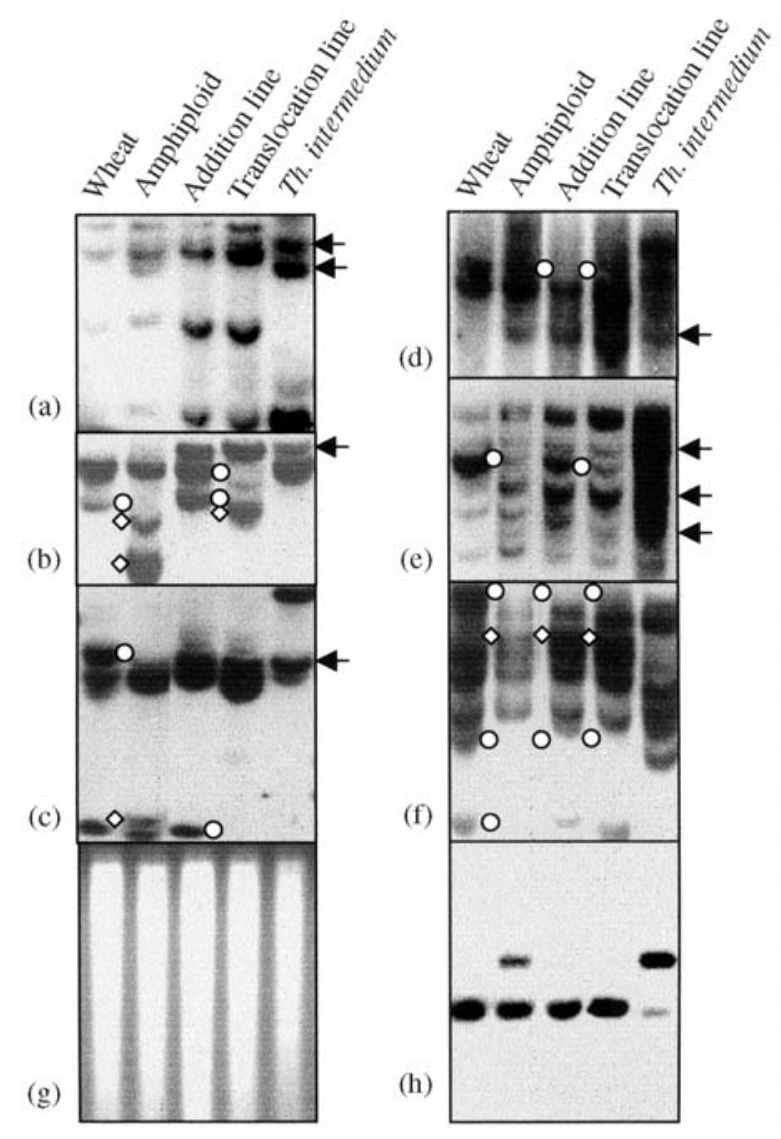

Figure 1 - Detection of the presence of Thinopyrum intermedium chromatin and genomic changes in octo-amphiploid (Zhong3), its derived addition line (TAI27) and translocation line (\#32). (a) Hybridization of probe HP80 (mapped to homoeologous group 7) to EcoRI-digested genomic DNAs of common wheat (lane 1), Zhong3 (lane 2), TAI27 (lane 3), Line \#32 (lane 4) and Th. intermedium (lane 5). The upper arrow denotes a Th. intermedium fragment present in all three lines (Zhong3, TAI27 and Line \#32) but the wheat parent; the lower arrow indicates a Th. intermedium fragment present only in Zong3. (b) Hybridization of probe Psr160 (mapped to homoeologous group 7) to EcoRI-digested genomic DNAs of the same samples as above. The arrow denotes a Th. intermedium fragment present only in TAI27 and line \#32; the circles and diamonds refer, respectively, to loss of wheat parental fragments and gain of novel bands in Zhong3 and Line \#32. (c) Hybridization of probe Psr148 (mapped to homoeologous group 7) to DraI-digested genomic DNAs of the same samples as above. The upper circle refers to loss of a wheat fragment from all three lines (Zhong3, TAI27 and Line \#32); the lower circle refers to loss of a wheat fragment from Line \#32. The diamond indicates gain of a novel fragment in Zong3. The arrow denotes a Th. intermedium fragment present in TAI27 and Line \#32. (d) Hybridization of probe CDO1164 (mapped to homoeologous group 3) to BamHI-digested genomic DNAs of the same samples as above. The arrow denotes a Th. intermedium fragment present in all three lines (Zong3, TAI27 and line \#32); the circles refer to loss of a wheat parental fragment in TAI27 and Line \#32. (e) Hybridization of probe Thg26 (a repeat isolated from genomic DNA of Th. intermedium) to EcoRI-digested genomic DNAs of the same samples as above. The arrows denote Th. intermedium fragments present in Zong3, TAI27 and line \#32; the circles refer to loss of a wheat parental fragment (or copy numbers thereof) from Zhong3 and Line \#32. (f) Hybridization of probe Psr170 (mapped to homoeologous group5) to EcoRI-digested genomic DNAs of the same samples as above. The circles and diamonds refer, respectively, to loss of wheat parental fragments and gain of novel fragments in Zhong3, TAI27 and Line \#32. (g) Ethidium bromide staining on digested DNA of the same samples as above, after agarose gel electrophoresis. (h) Hybridization to the same set of blots as above by a single-copy gene (encoding the putative sterol C24 methyltransferase, Genbank accession: AY226581).
Zhong3, cumulating to $47 \%$ at the genome-wide level, with the corresponding frequencies for addition line TAI27 and translocation line \#32 being, respectively, 43\% and 35\% (Table 1). The most frequent type of genomic change was fragment loss (disappearance of hybridization fragment), followed by loss/gain (simultaneous disappearance of fragment and appearance of new fragment), with gain (appearance of new fragment only) ranging last (Table 1 and Figure 1). Given that TAI27 and Line \#32 are direct derivatives of Zhong3, it seems odd that the incidences of genomic change in the addition and translocation lines were less frequent than that of amphiploid Zhong3. Moreover, as already mentioned above, additional introgression events, and hence probably other changes, also occurred subsequently to the formation of the addition line (Figures $1 b, c)$. The discrepancy could be reconciled if some of the genomic changes observed in Zhong3 had an epigenetic basis, such as alterations in DNA methylation patterns (Finnegan, 2002), which occurred in the amphiploid, but were not fully transmitted to the addition and translocation lines. Although most methylation changes in symmetric cytosines of plant genomes are highly heritable through meiosis, changes in asymmetric sites are less stable, and are likely to be lost during vertical transmission (Martienssen and Colot, 2001). In this regard, restriction sites of two of the enzymes used in the present study, BamHI and EcoRI, contain asymmetric cytosines known to be sensitive to a certain extent to methylation modifications. Thus, genomic changes due to methylation changes at these sites in amphiploid Zhong3 have probably contributed to the decrease in genomic changes detected in the addition and translocation lines (Table 1).

In contrast with the traditional view on polyploidy as being evolutionarily static, passive or even retardant (reviewed in Soltis and Soltis, 1995), recent work with several model polyploid plant species showed that nascent allopolyploidy could induce a rapid genome evolution that includes an array of genetic and epigenetic changes, which, although cannot be explained by Mendelian principles, are nevertheless potentially adaptive and evolutionarily consequential (reviewed in Leitch and Bennett, 1997; Matzke et al., 1999; Wendel, 2000; Comai, 2000; Pikaard, 2001; Liu and Wendel, 2002; Rieseberg, 2001; Levy and Feldman, 2002). The results of the present work appear to be in full accordance with the emerging view on allopolyploidy. Furthermore, our data suggest that human-made wide hybrids or allopolyploids are likely to be a rich source for cryptic introgressed novel plants that are potentially valuable materials for crop improvement.

\section{Acknowledgements}

This study was supported by the National Science Fund for Distinguished Young Scholars of China (30225003). We thank Professor MD Gale of the John Innes Center, Norwich, UK, for the wheat probes. 


\section{References}

Banks PM, Larkin PJ, Bariana HS, Lagudah ES, Appels R, Waterhouse PM, Brettell RIS, Chen X, Xu HJ, Xin ZY, Qian YT, Zhou XM, Cheng ZM and Zhou GH (1995) The use of cell culture for subchromosomal introgression of barley yellow dwarf virus resistance from Thinopyrum intermedium to wheat. Genome 38:395-405.

Brar DS and Khush GS (1997) Alien introgression in rice. Plant Mol Biol 35:35-47.

Chen Q, Conner RL, Li HJ, Sun SC, Ahmad F, Laroche A and Graf RJ (2003) Molecular cytogenetic discrimination and reaction to wheat streak mosaic virus and the wheat curl mite in Zhong series of wheat-Thinopyrum intermedium partial amphiploids. Genome 46:135-145.

Comai L (2000) Genetic and epigenetic interactions in allopolyploid plants. Plant Mol Biol 43:387-399.

Crasta OR, Francki MG, Bucholtz DB, Sharma HC, Zhang J, Wang RC, Ohm HW and Anderson JM (2000) Identification and characterization of wheat-wheatgrass translocation lines and localization of barley yellow dwarf virus resistance. Genome 43:698-706.

Fedak G, Chen Q, Conner RL, Laroche A, Petroski R and Armstrong KW (2000) Characterization of wheatThinopyrum partial amphiploids by meiotic analysis and genomic in situ hybridization. Genome 43:712-719.

Finnegan EJ (2002) Epialleles - a source of random variation in times of stress. Cur Opin Plant Biol 5:101-106.

Gale MD, Atlinson MD, Chinoy CN, Harcourt RL, Jia J, Li QY and Devos KM (1993) Genetic maps of hexaploid wheat. In: Li ZS \& Xin ZY (eds) Proc $8^{\text {th }}$ Int Wheat Genet Sympo. (pp 29-40). China Agri-Sci Tech Press, Beijing.

Han FP, He MY, Bu XL, Huang BQ, Hao S, Ma YZ and Xin ZY (1998) Characterization of a wheat-wheatgrass translocation line by FISH. Acta Bot Sin 40:500-502.

He MY, Xu ZY, Zhou MQ, Zhang H, Piao ZS, and Hao S (1988) The establishment of two sets of alien addition lines of wheat-wheatgrass. Sci Sin (ser. B) 32:695-705.

Larkin PJ, Banks PM, Lagudah ES, Appels R, Chen X, Xin ZY, Ohm HW and McIntosh R (1995) Disomic Thinopyrum intermedium addition lines in wheat with barley yellow dwarf virus resistance and rust resistance. Genome 38:385394.

Leitch IJ and Bennett MD (1997) Polyploidy in angiosperms. Trends Plant Sci 2:470-476.
Levy AA and Feldman M (2002) The impact of polyploidy on grass genome evolution. Plant Physiol 130:1587-1593.

Liu B, Luan YS, Han FP, Ji WQ and He MY (2001) Cell culture induced introgression of Thinopyrum intermedium chromatin into common wheat. Plant Cell, Tiss Org Cult 65:913.

Liu B, Segal G, Vega JM, Feldman M and Abbo S (1997) Isolation and characterization of chromosome-specific sequences from a chromosome arm genomic library of common wheat. Plant J 11:959-965.

Liu B and Wendel JF (2002) Non-Mendelian phenomena in allopolyploid genome evolution. Cur Genomics 3:489-506.

Martienssen RA and Colot V (2001) DNA methylation and epigenetic inheritance in plants and filamentous fungi. Science 293:1070-1074.

Matzke MA, Scheid OM and Matzke AJM (1999) Rapid structural and epigenetic changes in polyploid and aneuploid genomes. Bioessays 21:761-767.

Multani DS, Jena KK and Brar DS (1994) Development of monosomic alien addition lines and introgression of genes from Oryza-australiensis domin to cultivated rice Oryza-sativa L. Theor Appl Genet 88:102-109.

Pikaard CS (2001) Genomic change and gene silencing in polyploids. Trend Genet 17:675-677.

Rieseberg LH (2001) Polyploid evolution: Keeping the peace at genomic reunions. Cur Biol 11:R925-R928.

Soltis DE and Soltis PS (1995) The dynamic nature of polyploid genomes. Proc Natl Acad Sci USA 92:8089-8091.

Wang RR and Zhang XY (1996) Characterization of the translocated chromosome using fluorescence in situ hybridization and random amplified polymorphic DNA on two Triticum aestivum-Thinopyrum intermedium translocation lines resistant to wheat streak mosaic or barley yellow dwarf virus. Chromosome Res 4:583-587.

Wendel JF (2000) Genome evolution in polyploids. Plant Mol Biol 42:225-249.

Zamir D (2001) Improving plant breeding with exotic genetic libraries. Nat Rev Genet 2:983-989.

Zhang ZY, Xin ZY and Larkin PJ (2001) Molecular characterization of a Thinopyrum intermedium group 2 chromosome (2Ai-2) conferring resistance to barley yellow dwarf virus. Genome 44:1129-1135.

Editor Associado: Marcio de Castro Silva Filho 